\title{
Optimization of Mobile Applications in Mobile Cloud Computing (MCC)
}

\author{
Kuljeet Kaur ${ }^{1}$, Dr. Pankaj Deep Kaur ${ }^{2}$ \\ Master of Technology, Computer Science and Engineering, GNDU, RC, Jalandhar, India \\ Associate Professor, Computer Science and Engineering, GNDU, RC, Jalandhar, India
}

\begin{abstract}
Cloud computing is most important technique for providing applications on mobile devices. In this paper, cloud computing is introducing with better and efficient applications for mobile devices. It provides mobile users with data storage and processing services on a cloud computing platform. This paper discusses the cloud platform those are used for optimization of mobile applications and explain the issues of mobile cloud computing. The mobile applications are mainly implement in java, .Net etc.
\end{abstract}

Keywords: Mobile applications, Cloud Platform, Mobile Applications issues.

\section{INTRODUCTION}

Cloud computing gives a new idea of developing the mobile applications. Execution of any mobile application is not going to be dependent on handset with advance configuration any more.[1] Mobile application developers solve major challenges that are the existence of such a wide range of mobile operating systems. They are creating many versions of the same application. In any mobile device for any application execution two basic significant requirements are of processing power and memory of that device capable of supporting that corresponding application. Cloud Computing provides the opportunity to execute our applications on servers instead of running them locally and overcome the limitation of limited resources to a great extent. And also there will be no need for Mobile application developers to create many versions of same application. It's just the starting of a new phase of mobile application development there is still a long way to go to achieve a new mobile world infrastructure involving cloud computing in its base. [2].

Cloud computing is one of the most important technology which offers resource on demand facility. Cloud Computing is completely internet dependent technology. In this technology each client is assigned its own cloud with the help of different services like services of servers, storage, manage applications that can use by client [5]. Mobile cloud computing (MCC) brings new types of services and facilities for mobile users to take full advantages of cloud computing. The users require Saas, Paas or Iaas service which is provide through cloud. Mobile Cloud Computing at its simplest refers to an infrastructure where both the data storage and the data processing happen outside of the mobile device [15]. MCC is a service that concede resource constrained mobile users to adaptively adjust processing and storage capabilities by transparently partitioning and offloading the computationally intensive and storage demanding jobs on traditional cloud resources by arrange pervasive wireless access.[8] Mobile cloud applications measure the computing power and data storage away from mobile phones. [15]
The paper has been organized as follows: in section II describes the application of mobile cloud computing. In the section III explain the cloud platform of mobile cloud computing. In this section define the cloud platforms that are used for optimizing the mobile application. In the section IV issues of mobile cloud computing. The section $\mathrm{V}$ in describes the related work and next section VI in defines the future scope and next is the conclusion of this paper.

\section{APPLICATION OF MOBILE CLOUD COMPUTING}

Mobile computing is arrangement of applications on mobile devices. MCC accommodate mobile applications using cloud to give more power to mobile devices in the direction of computing [8]. Both hardware and software of mobile devices get greater improvement than before some smart phones such as iPhone 4S, Android serials, Windows Mobile serials and Blackberry, are no longer just traditional mobile phones with conversation, SMS, Email and website browser, but are daily necessities to users. [9] Some application of mobile cloud computing are:

\section{A. Mobile Commerce}

It is a business model. In mobile devices this model is used for commerce. The mobile commerce application completes some tasks like mobile payment, mobile transaction, mobile messaging, and mobile ticketing. [15]

\section{B. Mobile learning}

The main objective of Mobile-Learning is that the learners can get the knowledge from the centralized shared resources at any time. The users get the knowledge anywhere with the help of mobile learning and they like to read that too at free of cost. It is accomplished based on elearning and mobility. [16]

\section{Mobile healthcare}

M-healthcare offers hospitals and healthcare organizations variety of on-demand services on clouds rather than owning standalone applications on local servers. [15] 


\section{Biometric Application}

Biometric applications are devoted to fingerprint, face, or iris scanning, verification and identification systems. Mobile devices can deliver biometric affirmation back to the laboratory in order for it to be processed. Software dedicated to fingerprint, face, iris or any other biometric scanning which identifies and verifies individuals typically works in a laboratory setting where the client computer has unlimited access to the throughput and computational resources of the network. [8]

\section{E. Android Applications}

Android applications in some open source are included. First AndTweet is a popular open source Twitter client, and offload methods which send/receive network packets to/from the Twitter servers, using the ThinkAir framework. Second one of the most popular open sources is Android email clients, K-9 Mail that is look into the feasibility of offloading network intensive methods. [6]

\section{F. Social Network Application}

A cloud becomes a useful tool to help mobile users share photos and video clips efficiently and tag their friends in popular social networks as Twitter, Facebook, Line, skype, whats aap and hike etc. The mobile users are supported by several cloud services such as guiding their trip, showing maps, recording, and storing images and video. [9]

\section{G. Zompopo}

Zompopo is a context aware application that's tries to extend the capabilities of a generic calendar adding features. In this application through we can make calendar. [10]

\section{CLOUD PLATFORM FOR MOBILE APPLICATION OPTIMIZATION}

The mobile cloud application implement with the help of some frame work, tools and platform. In the MCC some application used web services some are used android platform. Mobile Applications are implement by java script, html etc. These applications for optimization use the platform like Google, Amazon EC2 etc.

Cloud Platforms are basically the hosts that devote the required resources (computational power, storage, Web access etc) to the client. It is an arrangement for executing software applications in a logically abstract environment comprising of various utility cloud services. Cloud platform is a platform which enables developers to write or design applications that run on cloud.

Cloud computing is being induced by cloud providers including Amazon, Google, Sales force and Yahoo as well as traditional vendors including Hewlett Packard, IBM, Intel, Microsoft and are approve by different users, ranging from an individual to large enterprises including General Electric. Few well-known cloud platforms are: [14]

- Amazon Elastic Cloud Computing (EC2)

- Google App Engine (GAE)
- Force.com

- Microsoft Azure

Think Air

Bakabas

\section{CHALLENGES}

In the field of MCC there are various challenges like handover delay, bandwidth limitation, task division for offloading, reliability, integrity of data delivered, and scalability. These issues of MCC without degradation in performance or change in infrastructure, security of data in mobile device within a cloud and in the communication channel, identity privacy, location privacy, etc. These issues are the biggest interferences in growth of mobile cloud computing [7]. In the mobile world using cloud computing concept is all about supplying mobile applications and services in the cloud. These applications approve through cloud service providers and then deliver it to end-users mobile handsets over the Internet when required. So in making remote applications available to mobile devices by the use of cloud computing. In MCC some extents of challenges are occur which are discussed here.

A. Limited Energy Source of Mobile Device

In the mobile devices power capacity is based on their batteries whose capacity is limited so it is very important to maximize the battery life. More and more application execution in the cloud means more battery saving but in general it is not possible to completely transfer the whole application execution to the cloud. If display element is taken under consideration then we can divide mobile application into two major categories, one is display applications and second is non-display application. For immersive applications, execution offload flexibility is even more constrained, as application functions running on server and device are tightly coupled. [10]

\section{B. Low Bandwidth}

Many research scholars has introduce the optimal and efficient way of bandwidth allocation or the bandwidth limitation is still a big concern because the number of mobile and cloud users is dramatically increasing. In case of rich internet and immersive mobile applications, e.g. online gaming, that require high-processing capacity and minimum network latency cloud computing faces challenges due to low bandwidth of mobile network. So an improved network bandwidth is required so that data transfer within the cloud and other devices can be improved.

\section{Data Delivery}

The feature of resource constrained, mobile devices such as PDAs in terms of memory, processing power, battery lifetime and screen size are vital point of concern. Data security is concerned organizations are needed to incorporate information assurance and operational security policies and procedures. Organization-wide training, education, and awareness package focusing on their issues can also be included to ensure that the policies and 
procedures are followed completely. Policies regarding access control, authentication procedures, account and user management, encryption, content assurance, and general communications security should be developed and compliance measures should be taken for enforcing them. It is very important to establish and maintain consumers ${ }^{\text {ee }}$ trust on to the mobile platform protection for providing user privacy and data/application secrecy from adversary. [11]

\section{High Computing Application}

Some applications may require massive processing power and storage, which cannot be deployed in mobile devices due to limited resources. Under such circumstances, we have to divide applications in such a way so that the complex part is done by a cloud and a simpler task is left to mobile device. [4]

\section{E. High Availability}

The ability of the cloud computing system to provide and support a large amount of different resources that are easily accessible and that are operating in optimal performance conditions for a predefined agreed amount of time. [12]

\section{F. Reliability}

The ability of the cloud computing system to perform and maintain providing its resources under unexpected failures, of e.g., storage, network connectivity and computing power, for a predefined amount of time.

\section{$G$. Division of application services}

In mobile cloud computing environment, due to the issue of limited resources, some applications of computeintensive and data-intensive cannot be deployed in mobile devices, or they may consume massive energy resources. Divide the applications and use the capacity of cloud computing to achieve those purposes, which is: the core computing task is processed by cloud, and those mobile devices are responsible for some simple tasks only. In this processing, the major issues affecting performance of mobile cloud computing are: data processing in data centre and mobile device, network handover delay, and data delivery time. [13]

\section{H. Privacy}

Privacy is one of the biggest challenges in the mobile cloud computing environment. Some applications which hire cloud computing store user's data remotely. Third party companies may sell this important information to some government agencies without the permission of the user. [3] Encryption provides most effective way to maintain integrity and confidentiality of information Encryption favours data storage and transport but it fundamentally prevents data processing. Therefore, initially it was quite useless to send encrypted data to cloud providers for processing.

I. Data Security and other Security Issues

Mobile devices are famous for malicious code. There are many chances to lose the data because mobile devices are mostly unprotected. An unauthorized person can easily access the information stored on the mobile devices. The top mobile threats that affect security are

- Data loss from lost/ stolen devices.

- Information stealing by mobile malware

- Data leakage through poorly written third party applications. [3]

\section{RELATED WORK}

Markus Schüring in 2011 gives an introduction into cloud computing and its different service models. Cloud computing services can be used by smart phones and other resource starved devices. The three different service levels have been analyzed with regard to mobile usage, where IaaS with its hardware oriented approach only seems to be appropriate for storage provisioning. The two other service levels, namely PaaS and SaaS seem to be of more interest, as they offer the possibility to run whole applications or parts of them in the cloud. Author covered different communication network architectures that can be used in order to support cloud computing services on smart phones and other resource-starved devices. Srinivas in 2012 describes the basic introduction of cloud computing. The strategies of cloud these strategies deploy in cloud computing. From a service or consumer point of view, there are essential usability, stability, and reliability problems to solve. Pragya gupta in September 2012 explained the Mobile Cloud Computing, as a development and extension of Cloud Computing and Mobile Computing, is the most emerging and well accepted technology with fast growth. The combination of cloud computing, wireless communication infrastructure, portable computing devices, location-based services, mobile Web etc has laid the foundation for the novel computing model. In this paper given an overview of Mobile Cloud Computing that includes architecture, benefits, key challenges, present research and open issues. Author define the services of cloud computing and define their uses in mobile cloud computing.

Sehoon Park in 2013 provides a highly efficient way of increasing the performance of mobile devices in the offloading architecture. The built-in proxy system detects heavy computational function codes from the out bound web servers by programmer annotations. Our offloading method Reduces the response time of Java Script based web applications and minimizes CPU utilization of mobile devices. This approach is even more useful for low-cost devices. It eliminates the hardware performance limitations of low-end devices, since the heavy loaded computational applications on the client side will be offloaded to the server side. The experimental result shows a considerable performance gain. The framework reduced the response time the turn-based gaming application. It provided an early constant performance, irrespective of the network bandwidth environment.

Amit K. Sharma in 2013 basic purpose of Mobile Cloud Computing is providing PC-liked services to mobile Terminals. However, as the existing different features 
between mobile devices and PCs, we cannot directly transplant the services from PCs' platform to mobile devices. Milos Stojmenovic in 2013 Author describe the biometric applications and define the how to use these application in mobile cloud and the cloud can secure to other users. The biometric applications improve the security issues in mobile. Lakshmi Neelima in 2014 investigates the concepts of Mobile Cloud Computing (MCC), challenging security issues and breaches, assorted subsisting security frameworks and conclusively some solutions that increase the security in the Mobile Cloud Environment. Most of the frameworks overlooked the security of utilize data privacy, data storage and energy preserving data sharing. Data privacy and mobile application that utilizes cloud are the most challenging factor.

Manmohan Chaturvedi gives overview the mobile cloud computing. The fallout of the proposed research is expected to be of interest to both E-governance and Ecommerce applications. The challenges in this evolving field of many research. First phase attempting to characterize the problem in formal terms and propose a lightweight mobile interface having limited dynamic capability. Abdullah Gani conclude that there are three main optimization approaches in MCC, which are focusing on the limitations of mobile devices, quality of communication, and division of applications services. With the high increasing of data computation in commerce and science, the capacity of data processing has been considered as a strategic resource in many countries. Mobile cloud computing (MCC) as a development and extension of mobile computing (MC) and cloud computing (CC) has inherited the high mobility and scalability. Hoang T. Dinh propose that Mobile cloud computing is one of mobile technology trends in the future since it combines the advantages of both mobile computing and cloud computing, thereby providing optimal services for mobile users. Author describes the applications supported by mobile cloud computing including mobile commerce, mobile learning, and mobile healthcare have been discussed which clearly show the applicability of the mobile cloud computing to a wide range of mobile services. Then, the issues and related approaches for mobile cloud computing have been discussed.

\section{FUTURE WORK}

In MCC some applications through security issues are improve in mobile cloud in device and in data. Android developers to actively participate and create new apps to extend the functionality of the product Developing an Android app requires proper training in Software development and those who can develop apps which suite the need of consumers can easily establish a successful career.

\section{CONCLUSION}

Implementation of cloud computing in mobile applications is going to be a trend in the future since it combines the advantages of both mobile computing and cloud computing, thereby providing optimal services for mobile users. According to Recent researches many mobile applications that will be executed through cloud computing. Here in this paper we have provided an overview of cloud platform, mobile applications and finally discussed about the challenges of implementing cloud computing in mobile applications. In this paper authors described the issues and some issues are improved and some are not properly remove

\section{ACKNOWLEDGMENT}

I am grateful to the almighty for his constant blessing. The paper publication would not be possible without the guidance of my teachers and constant support of my family and friends. So I would like to thanks my all near and dear ones for being there with me in my entire journey of research and publication.

\section{REFERENCES}

[1] Divya Narain, "ABI Research: "Mobile Cloud Computing the Next Big Thing", March 2009

[2] Armbrust, "Above the Clouds: A Berkeley View of Cloud Computing," 10 February 2009

[3] Srinivas, "Cloud computing basics" International Journal of Advanced Research in Computer and Communication Engineering, Vol. 1, Issue 5, July 2012

[4] Pragya gupta, "Mobile cloud computing: the future cloud", IIAREEIE, vol.1, issue 3, September 2012

[5] Hitesh A. Bheda, "Application Processing Approach for Smart Mobile Devices in Mobile Cloud Computing”, IJARCSSE, Volume 3, Issue 8, August 2013

[6] Miss Bhagyashiri D., "Development of android based Cloud Server for Efficient Implementation Platform as a Services", IJARCSSE, vol.4,issue 1, January 2014

[7] Manmohan Chaturvedi, "Privacy \& Security of Mobile Cloud Computing"

[8] Milos Stojmenovic, "Mobile Cloud Computing for Biometric Applications"

[9] Abdullah Gani, "Research on Mobile Cloud Computing: Review, Trend and Perspectives"

10] Jitendra Maan, "Extending the Principles of Cloud Computing in Mobile Domain" D.C. Wyld et al. (Eds.): NeCoM/WeST/WiMoN 2011, CCIS 197, pp. 197-203, 2011.C Springer-Verlag Berlin Heidelberg 2011

[11] Amit K. Sharma, "Mobile Cloud Computing (MCC): Open Research Issues", IJIET, Vol. 2 Issue 1 February 2013

[12] Markus Schüring, "Mobile Cloud Computing -open issues And Solution", 15thTwente Student Conference on IT, Enschede, The Netherlands, June 20th, 2011

[13] Lakshmi Neelima, "Mobile Cloud Computing: Issues from a Security Perspective", IJCSMC, Vol. 3, Issue. 5, May 2014

[14] Deepti Sahu, "Cloud Computing in Mobile Applications", International Journal of Scientific and Research Publications, Volume 2, Issue 8, August 20121 ISSN 2250-3153

[15] Chonho lee, "A Survey of Mobile cloud Computing: Architecture, Applications, and Approaches" wiely

[16] Mallikharjuna Rao, "Cloud Computing Through mobile learning" International Journal of Advanced Computer Science and Applications, Vol.1, No. 6, December 2010

\section{BIOGRAPHY}

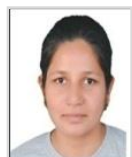

Kuljeet Kaur received Bachelor's Degree in Computer Science from Punjab Technical University, Jalandhar, India. She is pursuing her Master degree from Guru Nanak Dev University, Amritsar, India under the batch 2013-2015. Her interest Areas includes Cloud Computing. 